\title{
An Expeditious Enantioselective Synthesis of Antimycin $\mathbf{A}_{3 \mathbf{b}}$
}

\author{
Yikang $\mathrm{Wu}^{*}$ and Yong-Qing Yang \\ State Key Laboratory of Bio-organic \& Natural Products Chemistry, Shanghai Institute of Organic \\ Chemistry, Chinese Academy of Sciences, 354 Fenglin Road, Shanghai 200032, China \\ yikangwu@mail.sioc.ac.cn
}

\section{Table of Contents}

General remarks for Experimental Section
${ }^{1} \mathrm{H}$ NMR spectrum of 6
${ }^{1} \mathrm{H}$ NMR spectrum of 8
${ }^{13} \mathrm{C}$ NMR spectrum of 8
${ }^{1} \mathrm{H}$ NMR spectrum of 9

General remarks for Experimental Section: The anhydrous/reagents involved in the moisture/air sensitive experiments were dried as follows. THF: distilled from Na wire/ $\mathrm{Ph}_{2} \mathrm{CO}$ under $\mathrm{N}_{2}$ prior to use. $\mathrm{CH}_{2} \mathrm{Cl}_{2}$, pyridine, $\mathrm{NEt}_{3}$, TMEDA, and DMF: distillated over $\mathrm{CaH}_{2}$ under $\mathrm{N}_{2}$ prior to use. Column chromatography was performed with silica gel (0.063-0.2 mm). NMR spectra were recorded in $\mathrm{CDCl}_{3}$ on a spectrometer operating at $300 \mathrm{MHz}$ for proton. IR spectra were recorded on an FT-IR spectrometer. MS and HRMS experiments were performed on a high/low resolution magnetic sector mass spectrometer. Optical rotations were measured on a precision automated polarimeter. All yields given refer to as isolated yields. 

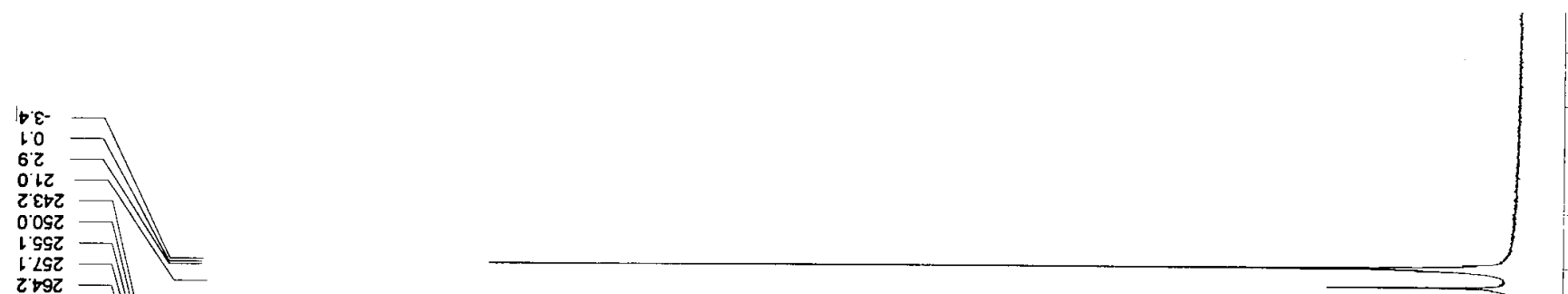

$\checkmark$ EQs

6.ZZ9

$8 \& Z 9$
$Z 6 Z 9$
$Z 0 \varepsilon 9$

วั0६9

c'ebs

c.etg

2099
$\square \quad 299$

\& 899

$\varepsilon .899$
$\varepsilon 108$

9508
2608

0.Z18

6218

5.918
$b 618$

ᄂ\&Z8
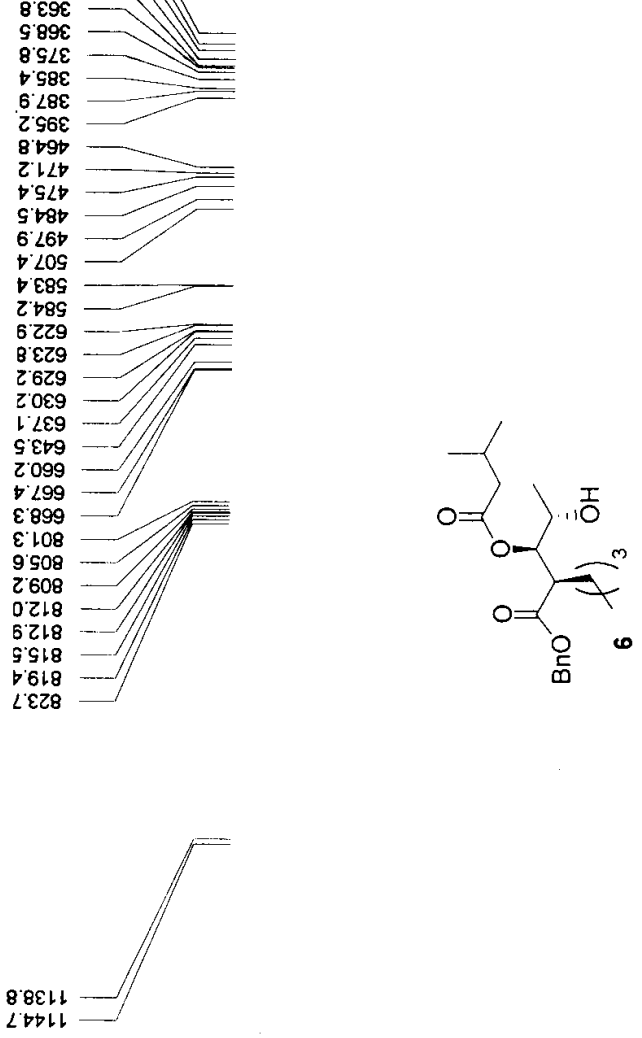

$\underset{L \not p L L}{88 \varepsilon l L}$
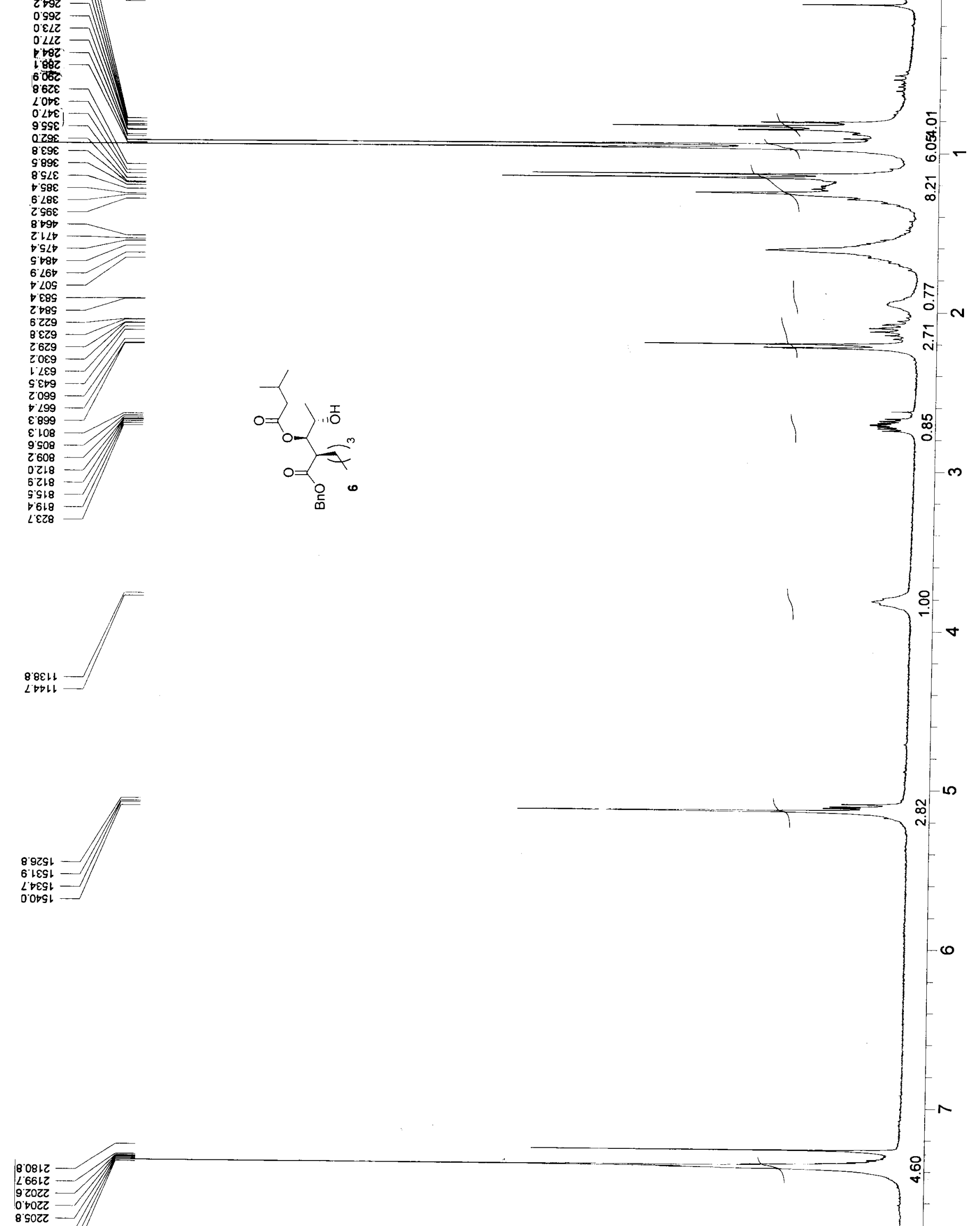

80812
$\angle 6612$

9 zozz

8 sozz

solzz 

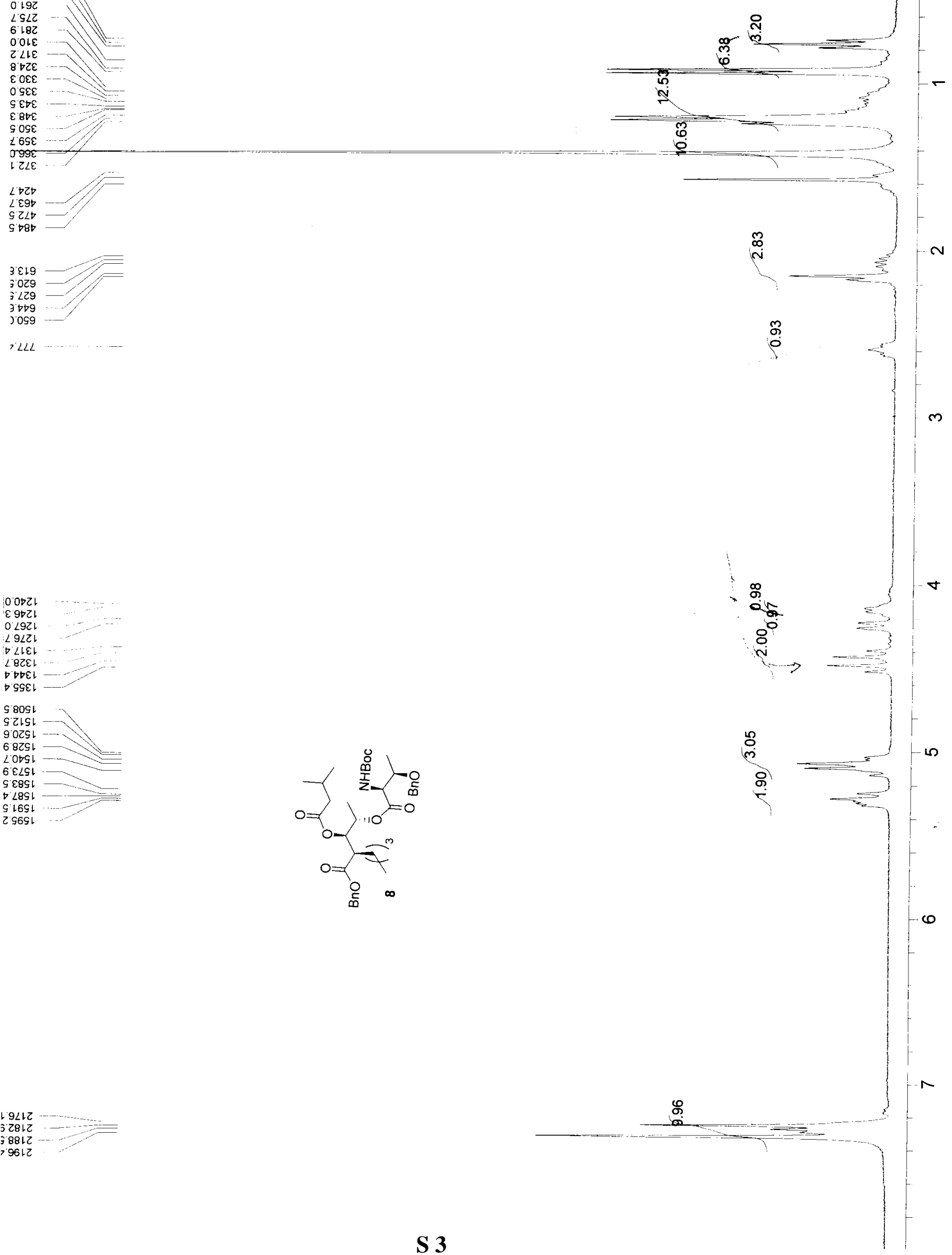

$\checkmark$ SGEL

S $809 \downarrow$

SZISL

90251
$68 Z 51$

68251
Lots

6 ELS।

SERS
$\rightarrow$ LBS

D. LSS
S. LG

2 S69

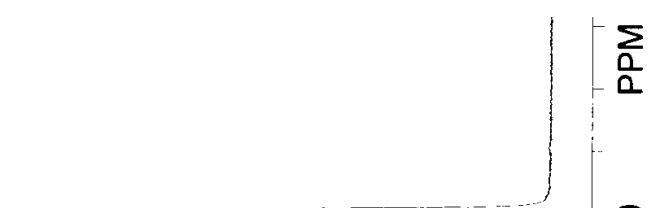

0
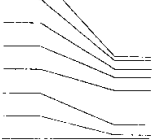
$9 Z L \cdot \varepsilon I$

$6 \varepsilon 6^{\circ} \circ \mathrm{I}$

$28 \varepsilon^{\circ} 91$

$\angle 6 Z \cdot 2 z$

$\varepsilon \angle b^{\circ} Z Z$

$\rightarrow 95^{\circ} \mathrm{s} \mathrm{Z}^{\circ}$

LI 22

$5 E Z^{\circ} 8 Z^{\circ}$

$060^{\circ} 62$

$\tau \angle z^{\circ} \varepsilon b$

$\angle D D^{\circ} 9 t$

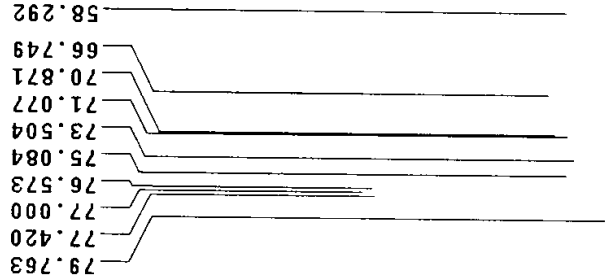

ISS $\angle Z T$

I $92 \cdot 82 \mathrm{I}$

乙E. $82 \mathrm{I}$

ISt $\mathrm{SEI}$

$660.8 \varepsilon \tau$

$286^{\circ}$ SSI

S90.0 $0<\mathrm{I}$

$2 b 60^{\circ} t<t$

$000 \cdot 221$

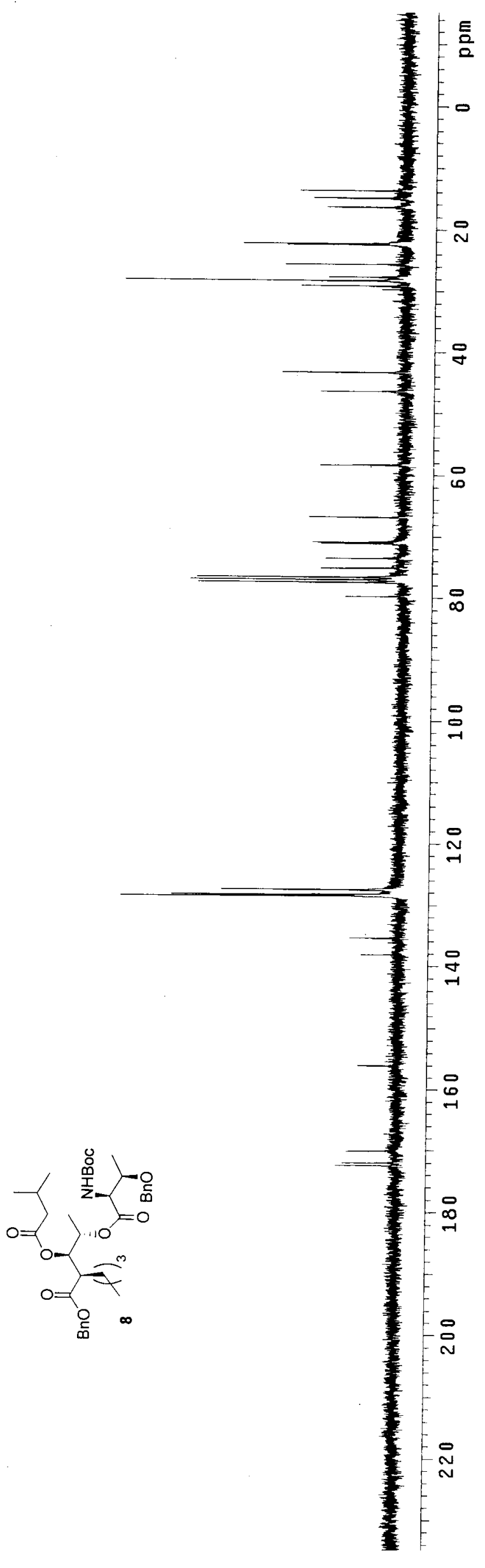




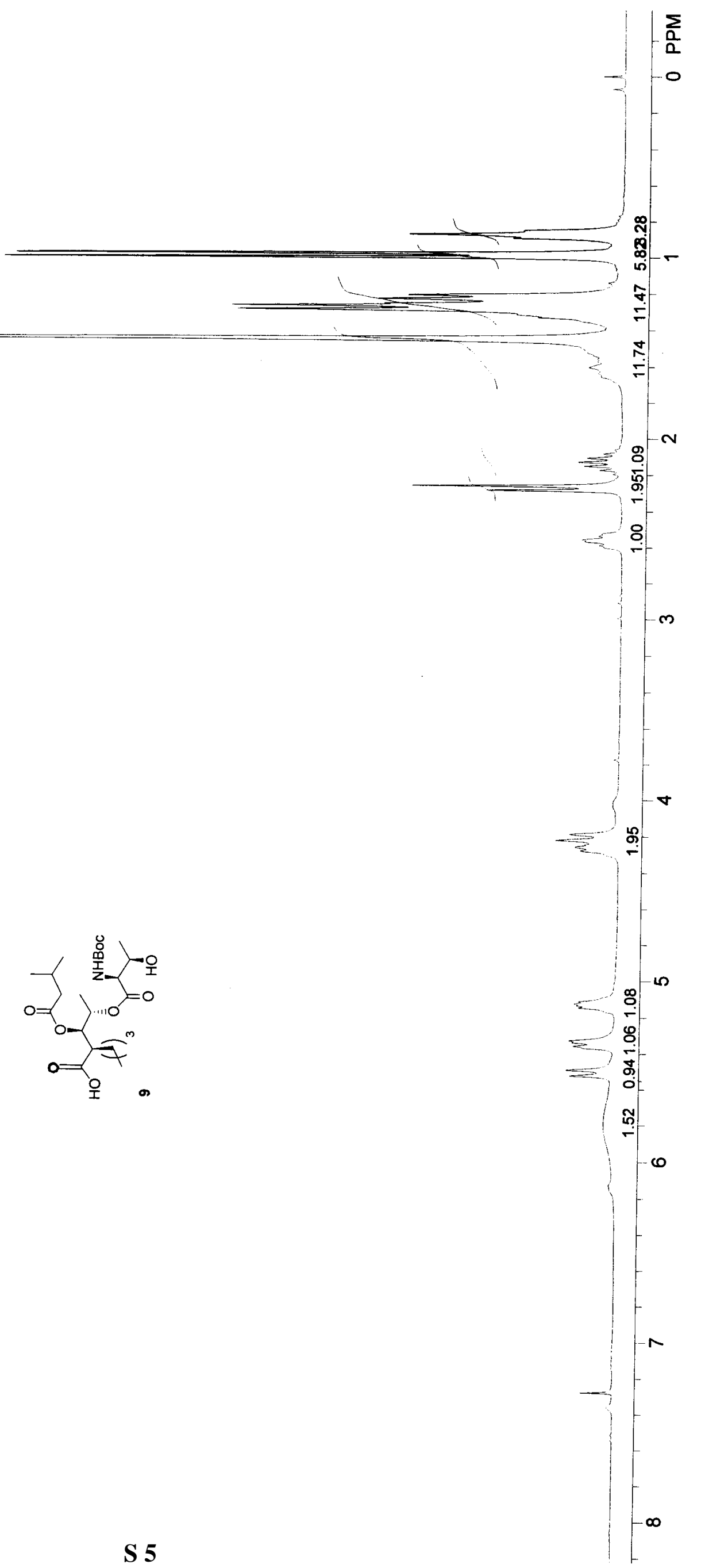




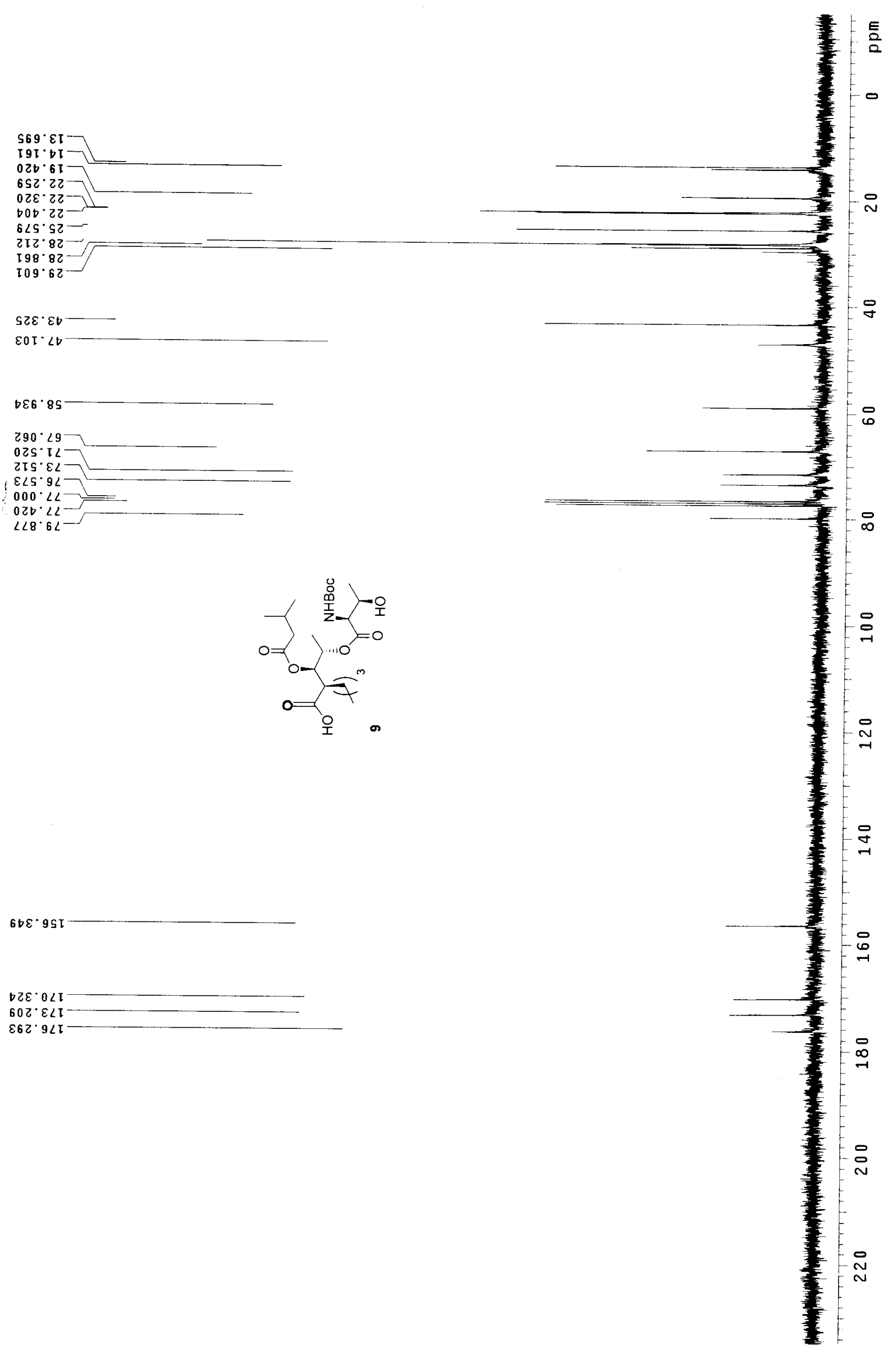


E6EL 6 Ib

9टЕ6 . 9ट०

$\triangle 898$ ' $E E \square$

D००6. 980

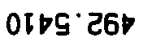

285I $\angle 29$

GEIE EEg

SG89. IDS

$O D \angle E^{\prime} 8 \nabla 9$

IOEB $\triangle 2 L$

โ682 $E L L$

IEOZ EI L

$6 \angle \angle 8^{\circ} 6$ I I

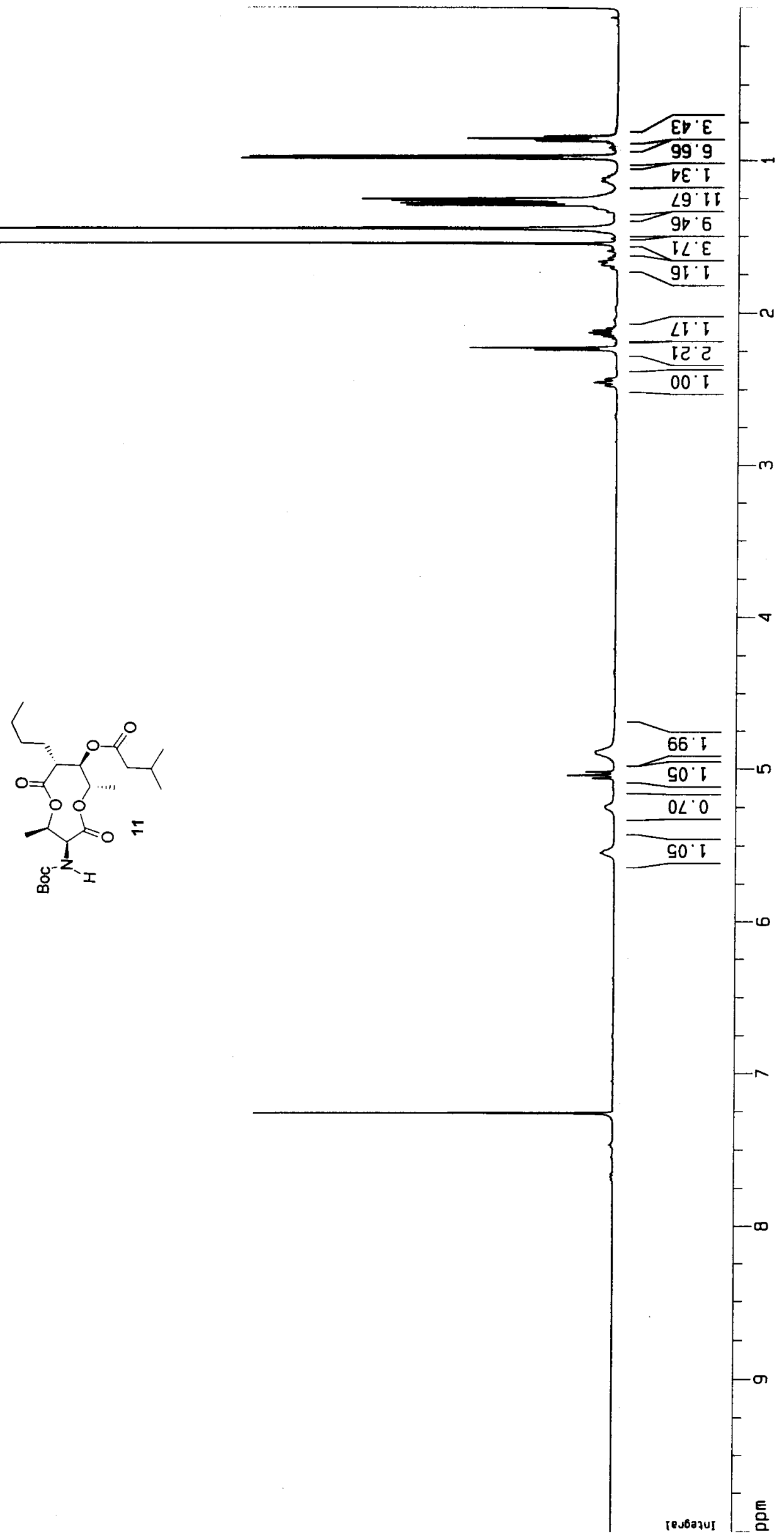

$68 \angle 8^{\circ} 0 \varepsilon 9 \varepsilon$ 
OSL $E$ L

$E E L \circ L$

$580^{\circ} \angle L$

S6E. टट

$090 \mathrm{ge}$

6rर 82

96I E己

999 6己

ODट ED

$180^{\circ} 09$

ع8० ०S

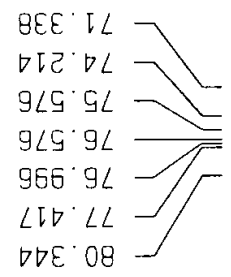

$800^{\circ} \circ 9$

$8 \varepsilon 9^{\circ} 0 \angle 1=$

$289^{\circ} \angle \angle L$

$998^{\circ} 2 \angle[-$

wdd

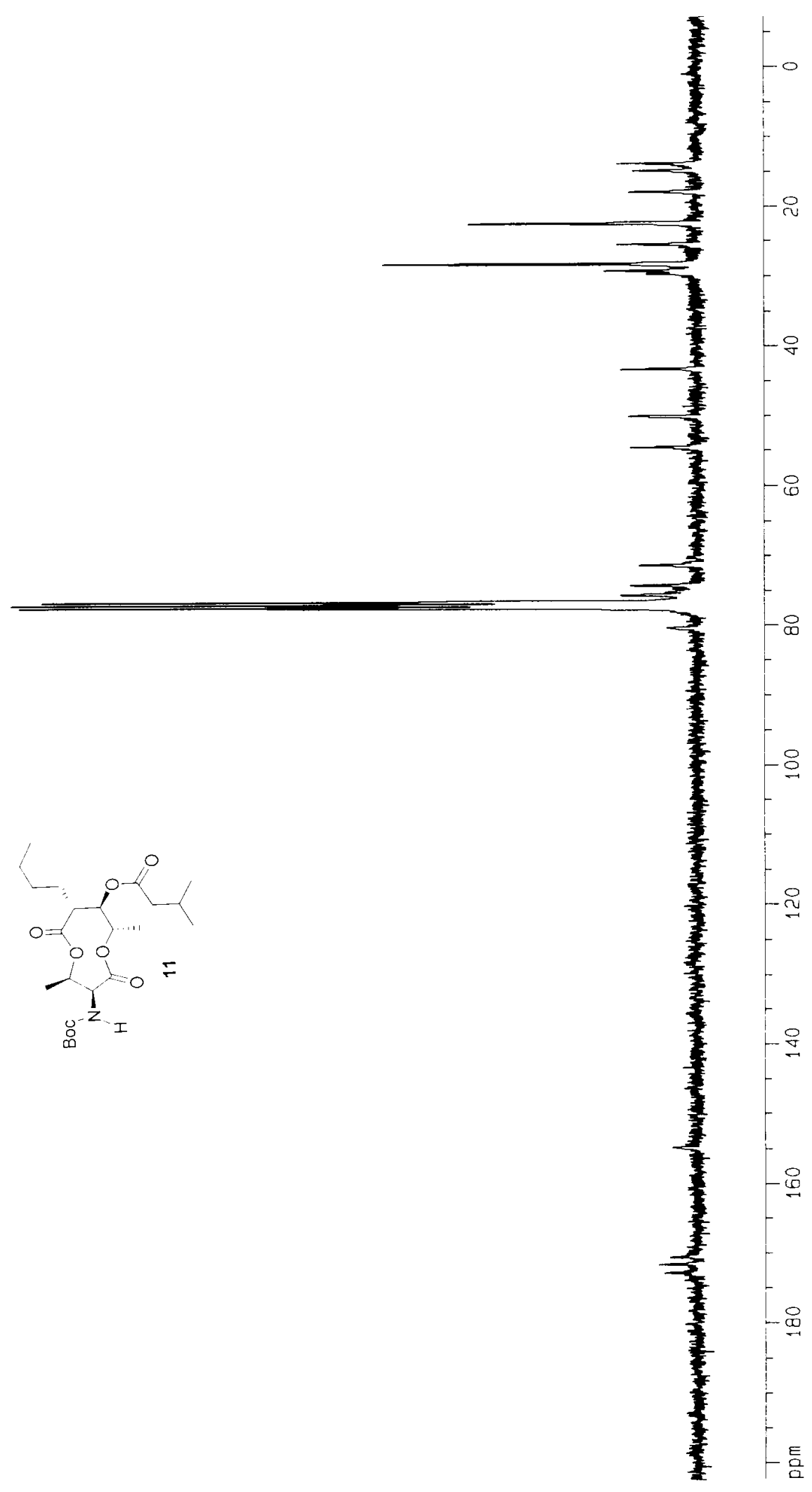


ZZsz -

2692

$6.992-$

1 isLz

6. 182

$8 \mathrm{\varepsilon bZ}$

0.918

0.928

$0.98 \varepsilon$

' 688

8.

$z 20 t$

8 top
2. 105

$\rightarrow \varepsilon+9$

$86+9$
$9.8<9$

$9 \varepsilon<9$
0.089

$06 \$ 2$

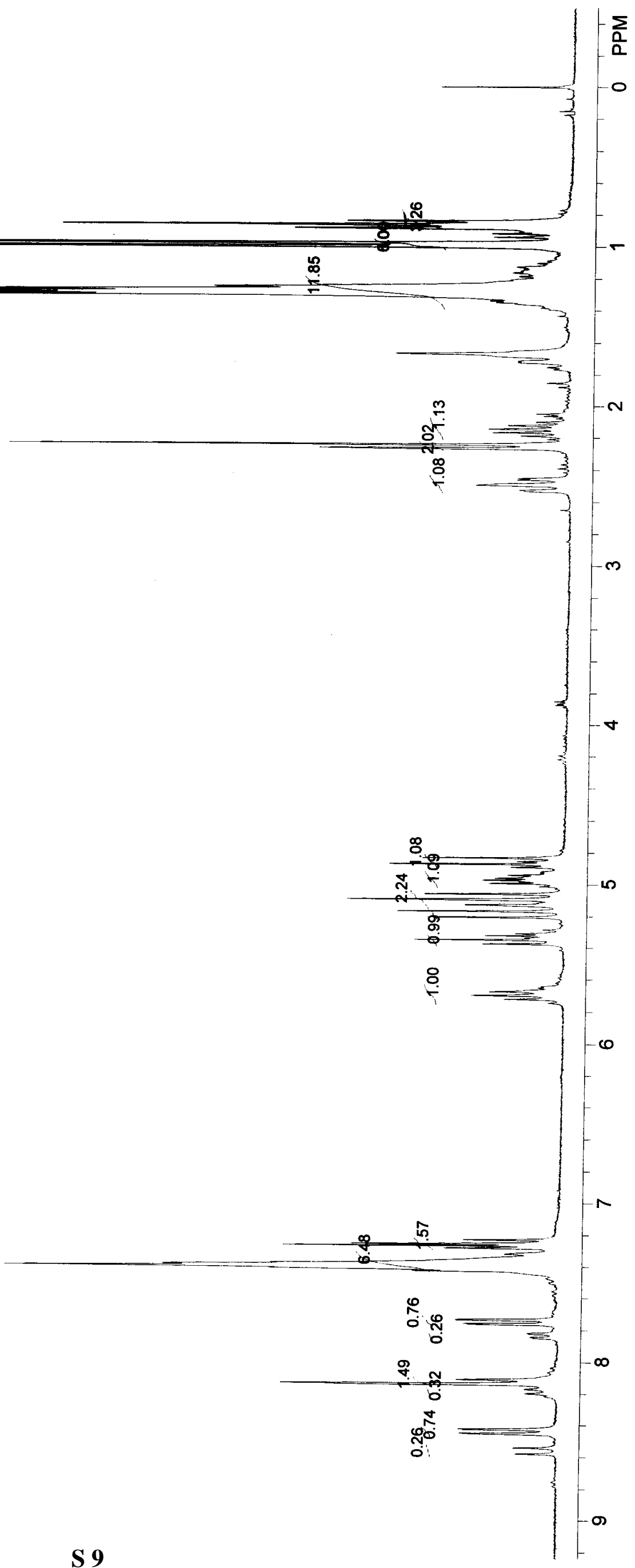

$960 p l$

$809 p 1$

$8.28 t$

s.t6tl- $=$

9 <6ㄴ

LLLIL

$08 \varepsilon 9$

toss

8 L. 9 L - -

L 265 L

z so9l

$=$

三

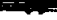
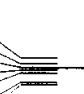

필

$\stackrel{\infty}{\infty}$

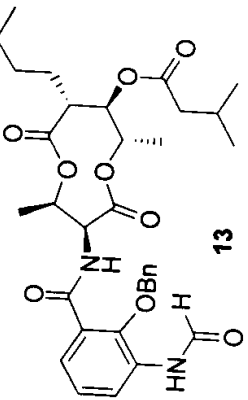

$960 \angle 1$

6891

Z LLL

losi

$808 \mathrm{~L}$

$z>812$
$0 \rightarrow 12 z$

olzz

8 'ozzz

$1+b z z 2$

$\varepsilon$ 'вzzz

861

乙LZ६

L

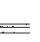

